\title{
Neutrino Oscillations at Proton Accelerators
}

\author{
Douglas Michael \\ California Institute of Technology \\ Pasadena, CA 91125 USA
}

\begin{abstract}
Data from many different experiments have started to build a first glimpse of the phenomenology associated with neutrino oscillations. Results on atmospheric and solar neutrinos are particularly clear while a third result from LSND suggests a possibly very complex oscillation phenomenology. As impressive as the results from current experiments are, it is clear that we are just getting started on a long-term experimental program to understand neutrino masses, mixings and the physics which produce them. A number of exciting fundamental physics possibilities exist, including that neutrino oscillations could demonstrate $\mathrm{CP}$ or CPT violation and could be tied to exotic high-energy phenomena including strings and extra dimensions. A complete exploration of oscillation phenomena demands many experiments, including those possible using neutrino beams produced at high energy proton accelerators. Most existing neutrino experiments are statistics limited even though they use gigantic detectors. High intensity proton beams are essential for producing the intense neutrino beams which we need for next generation neutrino oscillation experiments.
\end{abstract}

\section{OSCILLATION PHENOMENOLOGY}

Neutrino oscillations can arise if there is a non-exact alignment between flavor and mass eigenstates. Assuming three flavors and mass eigenstates, the most general mixing can be described by the following unitary transformation:

$$
\left(\begin{array}{c}
v_{e} \\
v_{\mu} \\
v_{\tau}
\end{array}\right)=\left(\begin{array}{ccc}
U_{e 1} & U_{e 2} & U_{e 3} \\
U_{\mu 1} & U_{\mu 2} & U_{\mu 3} \\
U_{\tau 1} & U_{\tau 2} & U_{\tau 3}
\end{array}\right)\left(\begin{array}{c}
v_{1} \\
v_{2} \\
v_{3}
\end{array}\right)
$$

The oscillation probability is then given by

$$
\begin{aligned}
& P(\alpha \rightarrow \beta)= \\
& \delta_{\alpha \beta}-4 \sum \sum U_{\alpha i} U_{\beta i} U_{\alpha j} U_{\beta j} \sin ^{2}\left(\left(m_{i}^{2}-m_{j}^{2}\right) L / 4 E\right)
\end{aligned}
$$

where $m_{i}$ are the mass eigenstates, $\mathrm{L}$ is the distance that the neutrino has propagated and $\mathrm{E}$ is the neutrino energy.

One can keep expanding this matrix to any arbitrary number of neutrino flavors and mass eigenstates to account for any number of possible sterile neutrinos or very massive neutrinos. Additional light "active" neutrinos (those that have normal couplings to the $Z^{0}$ ) are ruled out by results from LEP and SLC [1]. We also note that there is no a priori requirement that the neutrino and anti-neutrino matrices or masses must be identical. However, if they are different it requires CPT violation. It has been suggested that such violation is possible due to non-local string theories and/or that while CPT is exactly conserved in higher dimensions that in 4-dimensions the conservation is only approximate [2].

A specific form of the mixing matrix for the three known active flavors has been developed by Maki, Nakagawa and Sakata and is known as the "MNS matrix" [3]:
$U=\left(\begin{array}{ccc}c_{13} c_{12} & c_{13} s_{12} & s_{13} e^{-i \delta} \\ -c_{23} s_{12}-s_{13} s_{23} c_{12} e^{i \delta} & c_{23} c_{12}-s_{13} s_{23} s_{12} e^{i \delta} & c_{13} s_{23} \\ s_{23} s_{12}-s_{13} c_{23} c_{12} e^{i \delta} & -s_{23} c_{12}-s_{13} s_{23} s_{12} e^{i \delta} & c_{13} c_{23}\end{array}\right)$

Where $c_{i j}=\cos \theta_{i j}$ and $s_{i j}=\sin \theta_{i j}$. This is completely analogous to the CKM matrix for the quark sector. Of importance, we note that a CP-violating phase, $\delta$ is possible. Several authors have suggested that this phase may be measurable in future oscillation experiments $[4,5,6,7,8]$.

A final important feature of neutrino oscillation phenomenology are matter effects. When neutrinos propagate through matter the oscillations can change depending on the type of neutrinos involved. Space requires limiting discussion on this interesting point other than to note that matter effects may be important in considering future long-baseline neutrino oscillation experiments.

\section{REVIEW OF EXISTING OSCILLATION DATA}

Experimental evidence exists for three, apparently different, neutrino oscillation effects. The complete set of results now favors solar neutrino parameters with large (but not maximal) mixing and $\Delta m^{2}$ in the range of several $\times 10^{-5} \mathrm{eV}^{2}$. The atmospheric oscillation parameters show apparently maximal mixing and with $\Delta m^{2} \sim$ $3 \times 10^{-3} \mathrm{eV}^{2}$. The LSND oscillation parameters have very small mixing (less than $\sim 1 \%$ probability) and with $\Delta m^{2}$ in the range $0.1-1 \mathrm{eV}^{2}$. We note that if all of the experimental measurements and their errors are correct that just three active neutrino flavors provide only two $\Delta m^{2}$ values and hence cannot explain all of the experimental effects. However, all of the effects can be explained 




FIGURE 1. Compilation of allowed solar neutrino oscillation parameters. Only one region of parameter space remains at $90 \%$ CL. Adapted from reference [9].

if there are sterile neutrinos involved and/or if neutrinos and anti-neutrinos oscillate differently. A final observation is that neutrino masses suggested by the LSND $\Delta m^{2}$ can result in a significant (but not dominant) hot-darkmatter contribution by neutrinos in the universe.

\section{Solar Neutrinos}

In the last few years, data from Super-Kamiokande and SNO have significantly constrained the allowed solar neutrino parameters and phenomenology. The most important data for constraining the oscillation parameters comes from Super-Kamiokande. Super-Kamiokande observes no anomalous seasonal variation of the flux of neutrinos from the sun, observes an average flux of 0.45 that expected with no energy dependence and with no dependence on whether neutrinos have passed through the earth on their way from the sun to the detector or passed only through a small amount of overburden above the experiment (i.e. there is no "day/night" effect) [9]. The lack of anomalous seasonal variation rules out various "just-so" vacuum oscillation solutions. The lack of any modulation in the observed neutrino energy and the lack of any day/night effect constrains the oscillation parameters to relatively high mixing and $\Delta \mathrm{m}^{2}$ in the vicinity of several $\times 10^{-5} \mathrm{eV}^{2}$. Figure 1 shows the summary of the allowed solar oscillation parameters with data combined from both Super-Kamiokande and SNO along with older experiments.

The SNO data offers additional information compared to Super-Kamiokande due to the heavy water in SNO. Both Super-K and SNO measure some events resulting from elastic scattering of neutrinos off electrons in

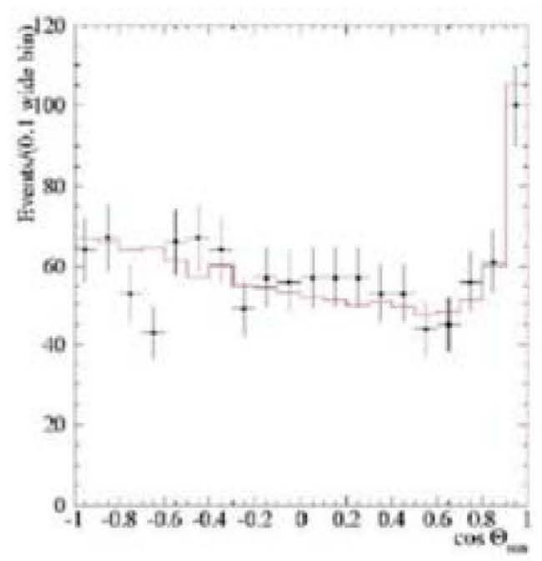

FIGURE 2. The observed rate of neutrino interactions in SNO vs the cosine of the angle from the sun along with a fit to the different types of neutrino interactions and background. Adapted from reference [10].

the water. This has both a charged and neutral current contribution. In SNO, there are two additional interactions: Charged-current dissociation of the the deuterium into two protons and an electron and the corresponding neutral current dissociation into a proton and a neutron. Figure 2 shows the observed rate of neutrino interactions in SNO vs the cosine of the angle from the sun. A contribution from elastic scattering of electrons is seen near $\cos \theta=1.0$, an overall normalization is visible resulting from a combination of $\mathrm{NC}$ deuterium dissociation and background and an overall slope, consistent with a $1-1 / 3 \cos \theta$ distribution is visible which results from the $\mathrm{CC}$ dissociation of deuterium. When combined with the results from Super-K on elastic scatters, one can determine with good precision both the total flux of neutrinos from the sun and the remaining flux of $v_{e}$ at the earth. The observed flux of all neutrino types of $5.44 \pm 0.99 \times 10^{6} \mathrm{~cm}^{-2} \mathrm{~s}^{-1}$ [10] is in impressive agreement with the standard solar model [11] value $5.44+1.01-0.81 \times 10^{6} \mathrm{~cm}^{-2} \mathrm{~s}^{-1}$. This results in a measured combined flux of $v_{\mu, \tau}$ of $3.7 \pm 1.1 \times 10^{6} \mathrm{~cm}^{-2} \mathrm{~s}^{-1}$.

The next important step in measurement of the "solar" neutrinos will come from the Kamland experiment, now taking data in Japan [12] Kamland is measuring the flux of $\overline{v_{e}}$ coming from several reactors in the vicinity of Kamioka with typical baseline distances between $100-$ $200 \mathrm{~km}$. Kamland should see a clear oscillation signature in these neutrinos if the suggested solar oscillation parameters are correct and if neutrinos and anti-neutrinos oscillate in the same way. The results from Kamland will be very interesting regardless of the outcome, but may imply different future experimental programs. Should Kamland not observe oscillations consistent with those suggested from the solar measurements then one possibility is that there is CPT violation in the neutrino sec- 
tor. This would mean that LSND could be the result of a higher $\Delta m^{2}$ for $\overline{v_{e}}$ than for $v_{e}$. It could also mean that there could be differences in $v_{\mu}$ and $\overline{v_{\mu}}$ oscillations with near atmospheric parameters and differences in the rate at which these may oscillate to $v_{e}$. On the other hand, it could mean that the solar oscillations are more subtle than currently supposed, or that some existing experimental results are incorrect. However, should Kamland see the expected oscillation signature at relatively high $\Delta m^{2}$ and large mixing then it is possible that future longbaseline oscillation experiments will be able to observe $\mathrm{CP}$ violation. We eagerly await the results of this experiment.

\section{Atmospheric Neutrinos}

The atmospheric neutrino data provides some of the strongest evidence that a classic oscillation effect is occurring in the neutrino sector (as opposed to any other means of neutrino flavor conversion). The data from Super-Kamiokande [13] are by far the most important for these measurements but there are also (pre)confirming data from MACRO, Soudan 2, Kamiokande and IMB [14].

The atmospheric oscillation effect is consistent with pure $v_{\mu}-v_{\tau}$ mixing and with that mixing being consistent between neutrinos and anti-neutrinos. However, the possibility of admixtures or differences in the oscillations still exist at the tens of percent level. Figure 3 shows the observed and predicted distributions of atmospheric neutrino events in Super-Kamiokande for $v_{\mu}$ and $v_{e}$. Several different observed energy regions are shown. The observed electron-like events are in good agreement with expectations with no oscillation while the observed muon-like events are in very poor agreement with a no oscillation hypothesis. However, the agreement with a simple $v_{\mu}-v_{\tau}$ oscillation model with $\Delta m^{2}=0.0028 \mathrm{eV}^{2}$ and $\sin ^{2} 2 \theta=1.0$ is excellent.

Accelerator-based long-baseline neutrino oscillation experiments are designed to study these oscillation effects with higher precision than the existing atmospheric data.

\section{LSND Oscillation Effect}

LSND (Los Alamos Scintillator Neutrino Detector) is the first accelerator experiment to report a positive observation of an apparent neutrino oscillation effect [15]. LSND observed the appearance of $\overline{\nu_{e}}$ in a $\overline{\nu_{\mu}}$ beam. Figure 4 shows the observed number of apparent $\overline{\nu_{e}}$ events compared to the expectations (from backgrounds) as a function of observed energy. The neutrino beam is produced from pions decaying at rest. This result suggests oscillation parameters as shown in figure 5. Since the so- lar neutrino $\Delta m^{2}$ is apparently much smaller, the parameters suggested from LSND must mean either that neutrinos and anti-neutrinos can have different $\Delta m^{2}$ or that there are sterile neutrinos involved (with large $\Delta m^{2}$ ) or that some experiments are wrong.

\section{NEAR-TERM OSCILLATION EXPERIMENTS AT PROTON ACCELERATORS}

Several experiments are either already running or in construction using accelerator neutrino beams to make further oscillation measurements. These experiments all already demonstrate the keen demand which neutrino oscillation experiments place on the intensity of high energy proton sources.

\section{Addressing the LSND Effect: Mini-BooNE}

The Mini-Boone (Mini-Booster Neutrino Experiment) [16] has just recently begun operation at Fermilab. It uses a neutrino beam generated from $8 \mathrm{GeV}$ protons from the Fermilab Booster. The nominal number of protons expected on target for the complete neutrino exposure is about $6 \times 10^{20}$. The recent discussion that neutrinos and anti-neutrinos may oscillate differently has also generated much interest in running with an anti-neutrino beam as well as the neutrino beam. This reduces the number of events by about a factor of three and hence for equal statistics requires roughly $18 \times 10^{20}$ protons on target.

The Mini-BooNE experiment uses a $1 \mathrm{KT}$ mineral oil scintillation/Cerenkov detector to observe neutrino events in the energy range from $200-2000 \mathrm{MeV}$. The baseline is about $300 \mathrm{~m}$. Figure 5 shows the allowed parameter space from the LSND and other measurements and the expected sensitivity for the Mini-Boone experiment in two years of running (for neutrinos). It is becoming fairly clear that a conclusive result may not be determined until the experiment has been able to achieve this exposure for both neutrinos and anti-neutrinos. With the nominally planned Booster intensity this could mean as much as 8 years of running. Clearly, a higher intensity of protons from the Booster is very desirable for this experiment.

\section{The first long baseline experiment: K2K}

The first "long-baseline" experiment, K2K, started operation in 1999. It utilizes the Super-Kamiokande detector and a beamline built for the $12 \mathrm{GeV}$ PS at KEK to send a neutrino beam $270 \mathrm{~km}$ through the earth to that detector. Although at $40 \mathrm{kT}$ the Super-K detector is easily the most massive available today for this type of measurement, the experiment is limited by statistical error 

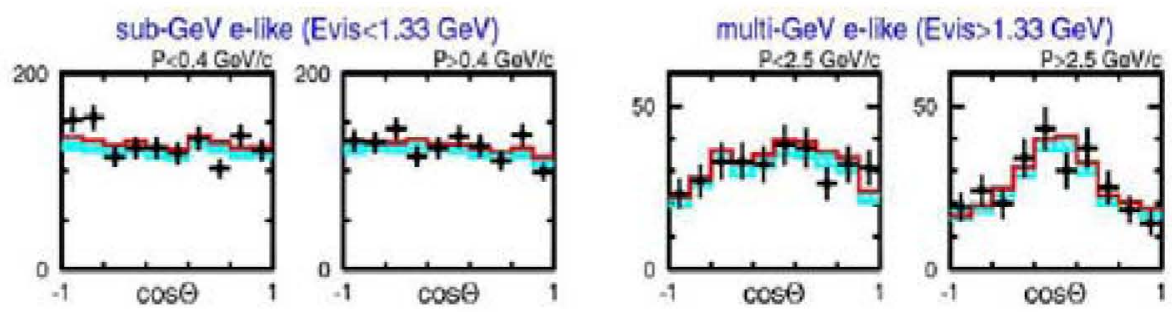

sub-GeV u-like (Evise1.33 GeV)
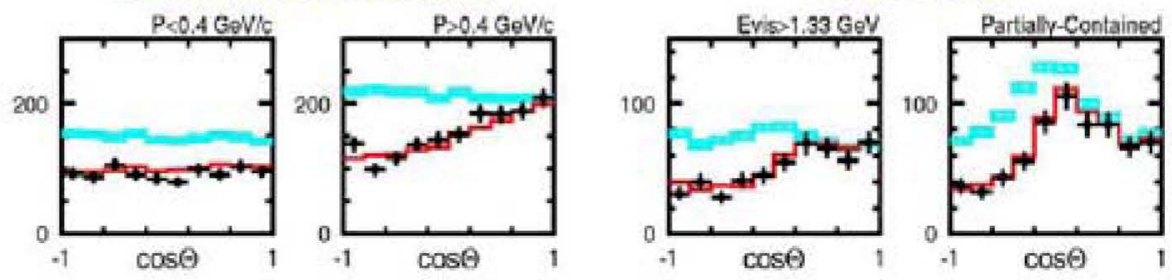

FIGURE 3. Observed and predicted atmospheric neutrino events in Super-Kamiokande. Adapted from [13].

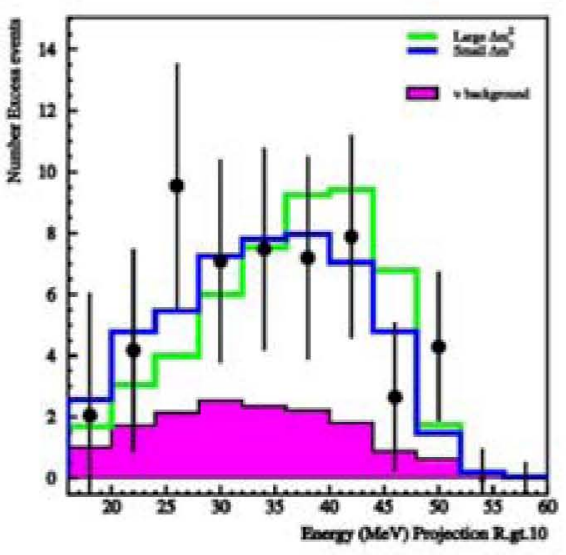

FIGURE 4. The observed number of $\bar{v}_{e}$ events in LSND vs neutrino energy compared to the expectation from backgrounds. Adapted from reference [15].

due to the relatively low intensity of the existing $12 \mathrm{GeV}$ machine. This may illustrate better than any existing experiment the relative importance of high-intensity proton accelerators as being a key element in the ability to make precise oscillation measurements.

$\mathrm{K} 2 \mathrm{~K}$ uses a "standard" neutrino beam configuration. The $12 \mathrm{GeV}$ protons are aimed in the direction (on axis) of Super-Kamiokande and then impinge on a target to produce pions. The pions are focussed using a doublehorn system and then directed down a decay pipe which is $\sim 150 \mathrm{~m}$ long. The peak neutrino energy is about 1.3 $\mathrm{GeV}$. To date, a total of $5.6 \times 10^{19}$ protons have been delivered to the $\mathrm{K} 2 \mathrm{~K}$ neutrino beam target. This has resulted in a total of 29 single-ring muon-like events observed at Super-Kamiokande with the expected number being $42 \pm 6$ events with no oscillations. An oscillation

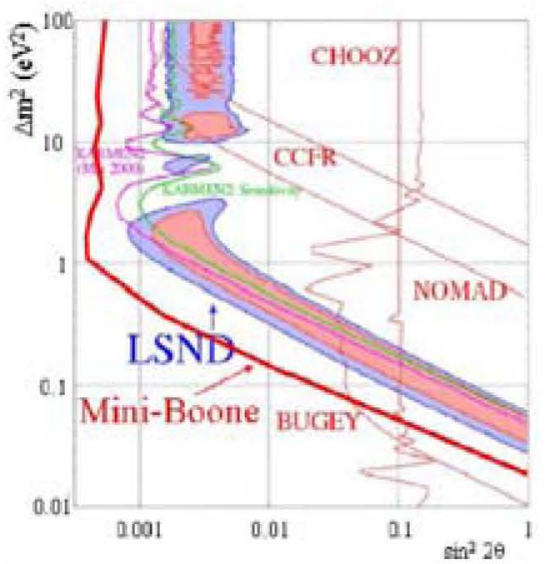

FIGURE 5. Allowed oscillation parameters for $\overline{v_{\mu}}$ to $\overline{v_{e}}$ based on LSND and other experiments. Mini-BooNE will clearly cover the allowed range of parameters.

analysis on these events shows that the resulting oscillation parameters are in good agreement with those measured from the atmospheric neutrinos [18].

\section{MINOS and the NuMI beamline at Fermilab}

The next step in precision in long-baseline experiments will come from the MINOS (Main Injector Neutrino Oscillation Search) experiment using the NuMI (Neutrinos at the Main Injector) beamline at Fermilab. MINOS will make use of the intense neutrino beam afforded by the Main Injector in order to make precise measurements on neutrino oscillations associated with the "atmospheric" neutrino oscillations. By 2005, the 
Main Injector will afford a very intense proton beam:

- $120 \mathrm{GeV}$ protons

- 1.9 s cycle time

- $4 \times 10^{13}$ protons per cycle

• $0.4 \mathrm{MW}$ !

- $3.6 \times 10^{20}$ protons per year.

The MINOS experiment uses a $735 \mathrm{~km}$ baseline and a peak neutrino energy of $3 \mathrm{GeV}$. Although the MINOS experiment is smaller than Super-Kamiokande (about a factor of 6 in fiducial mass) and the baseline is longer, the relatively large neutrino flux from the NuMI beamline more than compensates so the event rate in the MINOS far detector will be about 100 times that in SuperKamiokande with $\mathrm{K} 2 \mathrm{~K}$. This increase in neutrino flux permits better statistical precision and also qualitatively different types of measurements.

The NuMI beamline is very similar to that of the $\mathrm{K} 2 \mathrm{~K}$ beamline only somewhat larger in all dimensions. A graphite target is used due to the very high proton power. The energy of the neutrino beam can be tuned. A "low energy" beam is available with peak energy of $3 \mathrm{GeV}$.

The MINOS experiment utilizes a near and far detector of very similar construction in order to permit precision measurement of oscillation parameters, clear demonstration of the oscillation signature and precise determination of the flavor participation of all neutrino types involved in the oscillations. The MINOS detectors are sampling calorimeters with $2.54 \mathrm{~cm}$ thick iron absorbers interleaved with $1 \mathrm{~cm}$ thick plastic scintillator strips which are $4 \mathrm{~cm}$ wide. The iron in the detectors is magnetized with typical field of $1.5 \mathrm{~T}$.

The MINOS far detector has a total mass of $5.4 \mathrm{kT}$. Roughly $5000 v_{\mu}$ charged-current events are expected, in the absence of oscillations, in the far detector for two years of running. These statistics are sufficiently high that an unambiguous oscillation signature should be visible. Figure 6 shows the expected energy distribution of $v_{\mu}$ CC events measured in the MINOS far detector with and without oscillations for several different $\Delta m^{2}$ and $\sin ^{2} 2 \theta=0.9$.

In addition to measurement of the oscillation signature and parameters, MINOS will provide measurements of the participation of all types of neutrinos in the oscillations. By looking for appearance of $v_{e} \mathrm{CC}$ events in the far detector (or lack thereof) MINOS can determine the oscillation probability between $v_{\mu}$ and $v_{e}$ at the 1-2\% level. By comparing the number of NC events in the near and far detector MINOS can determine the level of oscillation of $v_{\mu}$ to $v_{\text {sterile }}$ at the several percent level. Neutrinos which disappear but do not oscillate to $v_{e}$ or $v_{\text {sterile }}$ must oscillate to $v_{\tau}$ so these are measured indirectly at the $10 \%$ level from the other measurements.
MINOS and the NuMI beamline are currently under construction. The first protons are scheduled on target in December 2004. The MINOS far detector is now half complete and the first half is being commissioned now for running for atmospheric neutrino measurements. The second half of MINOS is scheduled for completion by June 2003. Because of the magnetic field of MINOS, it will provide unique measurements on atmospheric neutrinos while awaiting completion of the NuMI beamline.

\section{OPERA, ICARUS and the CNGS beamline}

The final long-baseline project which is already in construction is the CERN to Gran Sasso (CNGS) beamline and experiments [17]. The CNGS beamline will use $400 \mathrm{GeV}$ protons from the SPS to create a high energy neutrino beam $\left(E_{\text {avg }}=17 \mathrm{GeV}\right)$ aimed at the Gran Sasso. The initial primary goal of this beamline and the experiments at the Gran Sasso will be to demonstrate direct appearance of $v_{\tau}$ CC events in the nominally $v_{\mu}$ beam. This will be possible in these experiments due to the combination of the high energy beam and large mass, very finegrained detectors which are capable of identifying the $v_{\tau}$ events. This beam will commissioned in 2006 .

The two detectors which will initially utilize this beam at the Gran Sasso are Opera and Icarus. Opera will use plates of emulsion interleaved with thin lead plates to look for CC $v_{\tau}$ events where the $\tau$ is produced in the lead and then subsequently decays in a gap downstream where a kinked track will be observed in the emulsion. Opera plans a total detector mass of about $2 \mathrm{kT}$, dominated by the mass of the lead. Icarus is a large liquid argon TPC which will use techniques similar to those used by the NOMAD short-baseline oscillation experiment to identify CC $v_{\tau}$ events on a statistical basis through the transverse momentum imbalance which results when a $\tau$ decays to a muon or electron. Icarus is currently building and commissioning a $600 \mathrm{~T}$ detector and plans for a $3000 \mathrm{~T}$ detector by the time the CERN beam is commissioned. These detectors will have similar sensitivity to $\tau$ appearance with about 10 events expected in 5 years of operation with $<1$ event background.

\section{NEXT GENERATION OSCILLATION EXPERIMENTS AT PROTON ACCELERATORS}

Even as the first round of very challenging long-baseline experiments is still under construction, already plans are developing for next-generation experiments. The current plans are primarily driven by the desire to search for a very small fraction of $v_{\mu}$ to $v_{e}$ oscillation (perhaps at the $1 \%$ level?). Once such an oscillation is identified the goal will be to search for whether there is a difference be- 

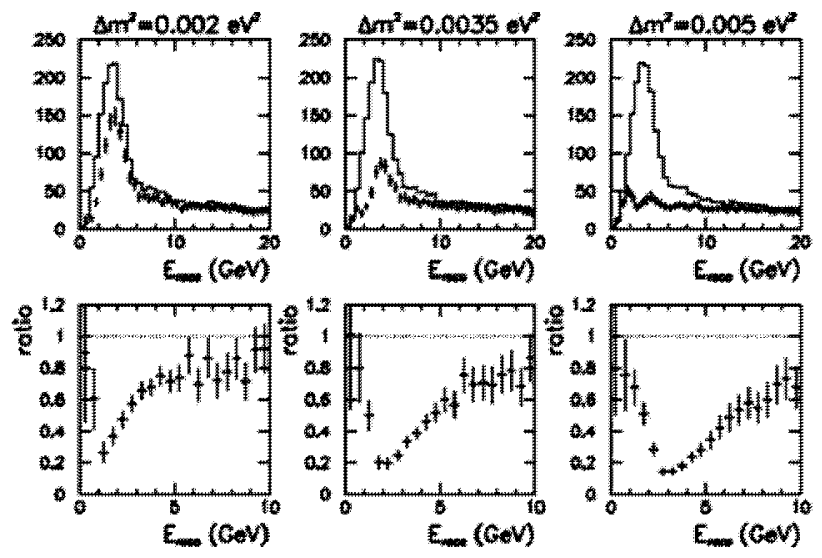

FIGURE 6. The top row of plots shows the expected energy distribution of $v_{\mu}$ CC events measured in the MINOS far detector with and without oscillations for several different $\Delta m^{2}$ and $\sin ^{2} 2 \theta=0.9$. The bottom row of plots shows the ratio of the spectra with and without oscillations. A clear dip in the ratio of neutrino energies with oscillations compared to the unoscillated spectrum will be observed within the expected range of $\Delta m^{2}$.

tween neutrinos and anti-neutrinos in these oscillations, resulting from $\mathrm{CP}$ or $\mathrm{CPT}$ violation or some combination of the two. Several theory papers in the last year have suggested that in fact this kind of subtlety in neutrino oscillations could exist $[4,5,6,7,8]$.

In the previous section, we have seen that with existing detectors, accelerators and neutrino beam facilities that we are already pushing hard on technology to start to make precise measurements on oscillations. Taking the next step will require both larger, more-sensitive detectors and higher fluxes of neutrino beams, driven by more intense beams of protons.

\section{Superbeams: The next step?}

A number of ideas have been discussed for future high-intensity neutrino beams. However, it appears that a consensus has developed over the last couple of years that the next step in long-baseline oscillation physics will be built around "Superbeams". The term "Superbeam" is somewhat poorly defined, and yet most people have some idea what it means. The term generally refers to a neutrino beam of standard, or nearly standard construction but with a very intense proton source and where the beam may be used in somewhat novel ways compared to existing experiments. Most of these plans call for use of "off-axis" neutrino beams (discussed further below).

For future experiments, total proton beam power in the range of $\sim 1-4 \mathrm{MW}$ are generally discussed. This is based in part on the capabilities of proton accelerators that are either being designed or planned. It is also based in part on a minimization of the total cost for future experiments, regardless of any explicit proton accelerator design. Generally, it is realized that once one will be spending on order $\$ 100 \mathrm{M}$ or more for gigantic detectors that equivalent investment in proton acceleration capabilities is likely to offer comparable value to the ultimate neutrino measurement capabilities. In general, both very large detector mass and the highest possible proton power will be necessary for the best sensitivity.

New high-intensity long-baseline experiments have been proposed for CERN, JHF, Brookhaven and Fermilab. Here, some of the possibilities are illustrated with the JHF to Super-Kamiokande proposal and the proposal for an off-axis experiment using an enhanced NuMI beam.

\section{The JHF neutrino superbeam to Super-K/Hyper-K}

A new $50 \mathrm{GeV}$ proton synchrotron is under construction at Tokai-mura, Japan. The new facility is generally referred to (in neutrino circles at least) as the "Japanese Hadron Facility", JHF. The first-phase plans for this machine call for a cycle rate of $0.29 \mathrm{~Hz}$ and for a total of $3.3 \times 10^{14}$ protons to be accelerated to $50 \mathrm{GeV}$ every cycle, corresponding to a total power of $0.77 \mathrm{MW}$. In addition, the plans call for an off-axis beam with a novel means of tuning the beam energy given that the far detector location is already determined by the location of the existing Super-Kamiokande detector. As with other "superbeam" experiments currently being planned, the primary goal for this experiment is to search for $v_{e}$ appearance, and if observed to compare with $\bar{v}_{e}$ appearance to search for $\mathrm{CP}$ and/or CPT violation. Due to shortness of space and because this experiment is described in more detail elsewhere in these proceedings [19], we curtail further description here. 


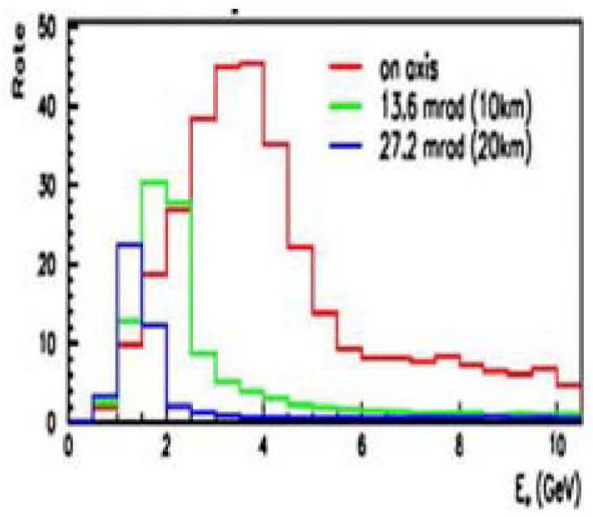

FIGURE 7. The rate of neutrino events vs neutrino energy for the NuMI on-axis and two off-axis beams.
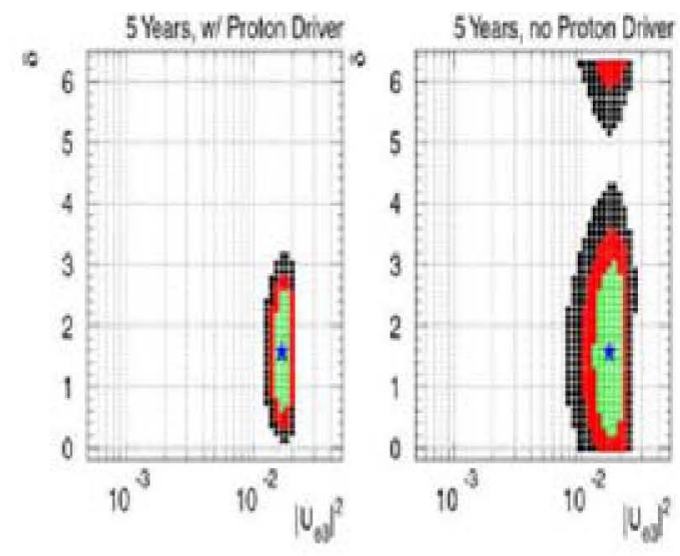

FIGURE 8. Comparison of possible $\mathrm{CP}$ violation measurements with a $20 \mathrm{kT}$ off-axis experiment using the NuMI beamline with and without a new proton driver. $\delta$ is the CP violating phase and $U_{e 3}^{2}$ is the relevant mixing angle for $v_{e}$ appearance. The measurement contours for 1,2 and $3 \sigma$ are shown for fi ve years of running (sharing between $v$ and $\bar{v}$ running) with a new proton driver on the left and without a new proton driver on the right. Although the oscillation parameters are unlikely to be exactly as shown here, the relative capabilities for precise measurements is similar for a wide range of parameters. Adapted from $[20,21]$.

\section{Bulking up the NuMI beamline: From Intense to Super and off-axis}

As noted above, the NuMI beamline will already have $0.4 \mathrm{MW}$ of proton power starting in 2005. If not already a "super-beam", then it certainly is already well on its way. Recently new studies have been made for possible upgrades to the NuMI beamline and a new off-axis experiment. Part of the planning is to consider upgrades to the proton intensity through the Main Injector and upgrades to the NuMI beamline.

Figure 7 illustrates why off axis beams are of particular interest in general for measurement of $v_{e}$ appearance and specifically shows the case for the NuMI off-axis beam [21]. The rate of neutrino events vs neutrino energy per kT-year is shown at the distance of $735 \mathrm{~km}$ from Fermilab for the on-axis and two off-axis beams, one at $13.6 \mathrm{mR}$ and one at $27.2 \mathrm{mR}$. One observes that although the total rate of neutrino events is lower for the off-axis locations that the rate at certain low neutrino energies is higher than for the on-axis location. Of additional importance for $v_{e}$ appearance is that the off-axis beams have relatively less high-energy tail which can contribute background, primarily from NC single $\pi^{0}$ events.

Several experimental concepts have been considered for a new off-axis experiment in the NuMI beamline $[21,22,23]$. Some of the technologies which are being considered include sampling calorimeters with a variety of absorbers and active elements and liquid argon. The sampling calorimeters generally attempt to reduce the average $\mathrm{Z}$ of the detector components from those in MINOS (dominated by the 1 inch thick iron plates) and provide finer longitudinal and transverse sampling. It is expected that these improvements will reduce the background from NC $\pi^{0}$ events to well below that from the intrinsic $v_{e}$ 's in the beam. Is is expected that detectors with mass around $20 \mathrm{kT}$ will provide good initial measurement capabilities.

Ultimately, the goal is not to simply measure that there is some small admixture of $v_{\mu}$ to $v_{e}$ oscillation but to measure whether there is $\mathrm{CP}$ violation in the neutrino sector. Since this requires running with anti-neutrinos as well as neutrinos and looking for relatively subtle differences between the two on an already small signal... it is clear that one will wish to combine both the largest affordable detector and the most intense beam possible.

There are two, partially overlapping paths to providing higher intensity beams for the NuMI beamline. First, investments can be made in the existing accelerator complex, particularly in the Booster and Main Injector to increase the number of protons which can be delivered. Some modest improvements are already underway to meet the needs for the Collider Run II B and for MINOS and Mini-BooNE. Additional investment possibilities in the existing complex have been identified which could result in as much as a factor of two increase in intensity compared to the nominal NuMI design [24]. To make even greater increases in the intensity, Fermilab has also been studying the possibility of replacing the existing Linac and Booster with a new proton source, either a new $8 \mathrm{GeV}$ synchrotron or a new $8 \mathrm{GeV}$ LINAC. Although more expensive than simply investing in the existing accelerator complex, these machines, coupled with investments in the Main Injector (which overlap in any of the cases) could bring the total proton beam power for the NuMI beamline to 2-4 MW [25]. Such intense beams would likely require additional shielding upgrades in the target region of the current NuMI beamline. 
Should a new proton driver become available at Fermilab, the potential for very precise neutrino oscillation measurements exists. Figure 8 shows the relative precision of $\mathrm{CP}$ violation measurement that might be possible using a new proton driver compared to the measurement possible simply using the nominal design flux for the NuMI beam. It is clear that impressive precision can be reached with appropriate upgrades to the "existing" experimental complex.

\section{THE LONG-TERM FUTURE}

It is generally expected that in the long-term that neutrino beams generated from muon storage rings will provide the best oscillation measurements. Such beams will have unique properties:

- Very intense beams, on order tens to hundreds of times more intense than planned superbeams.

- Relatively clean narrow-band beams, though measurement of charge in detectors is important since the starting beams will simultaneously consist equal numbers of $v_{\mu}$ and $\bar{v}_{e}$ or $\overline{v_{\mu}}$ and $v_{e}$.

- Intense, high energy $v_{e}$ and $\bar{v}_{e}$ beams! At the same time as it leads to specific detector requirements, these beams also add very significant measurement capabilities achievable in no other way.

Because there are a number of technical issues to be addressed, some of which are further developed for the super-beams, it is now generally realized that experiments based on these muon storage ring beams will begin sometime well into the next decade at the earliest.

\section{CONCLUSIONS}

Neutrino oscillations present an ever more compelling avenue towards deeper understanding of fundamental physics beyond the standard model. The oscillation phenomenology can be complex and very interesting. What we have already learned about oscillations has required a wide array of different types of experiments and detectors, including accelerator based experiments. Our future understanding of oscillations will continue to depend on a wide array of experiments, and even more on measurements in long-baseline accelerator experiments. We are just getting started on a series of precision measurements which high-energy accelerator experiments can offer. There are many exciting prospects and specific projects being defined. One of the major themes which unites all of these projects is the need for very intense proton beams for production of the intense neutrino beams which are essential to the precision future experiments. The future of neutrino physics rests on our ability to generate, control and utilize these beams.

\section{REFERENCES}

1. Many measurements... See in example M. Akrawy etal, Phys.Lett.B231:530,1989.

2. G. Barenboim, J. Beacom, L. Borrisov, B. Kayser, Phys.Lett.B537:227,2002.

3. Z. Maki, M. Hakagawa, S. Sakata, Prog.Theor.Phys.28:870,1962.

4. Probing CPT Violation with Atmospheric Neutrinos, S. Skadhauge, hep-ph0112189.

5. Neutrinos as the Messenger of CPT Violation, G. Barenboim, L. Borissov, J. Lykken, A. Smirnov, Submitted to Phys.Rev.Let. hep-ph/0108199.

6. CPT Violation and the Nature of Neutrinos, G. Barenboim, J. Beacom, L. Borissov, B. Kayser, hep$\mathrm{ph} / 0203261$.

7. Neutrinos that violate CPT and the Experiments That Love Them, G. Barenboim, L. Borissov, J. Lykken, hep-ph/0201080.

8. Interpreting the LSND Anomaly: Sterile Neutrinos of CPT Violation or...?, A. Strumia, hep-ph/0201134.

9. Super-Kamiokande Collaboration, presented by M. Smy at Neutrino 2002, 'Super-Kamiokande's solar neutrino results", Munich, May 2002.

10. The SNO Collaboration, Presented by A. McDonald at TAUP 2001, Gran Sasso, Italy, Sep. 2001.

11. J. Bahcall, M. Pinsonneault, S. Basu, Astophys.J.555:990, 2001.

12. J. Shirai for the Kamland Collaboration, Presented at Neutrino 2002, Munich, Germany, May 2002.

13. Most recent results presented by M. Shiozawa at Neutrino 2002, Munich, Germany, May 2002.

14. Most recent results presented by M. Goodman at Neutrino 2002, Munich, Germany, May 2002.

15. C. Athanassopoulos etal., Phys.Rev.Lett.81:1774,1998.

16. "The Mini-BooNE Experiment", Presented by R. Tayloe at Neutrino 2002, Munich, Germany, May 2002.

17. For recent status see talk by S. Katsenevas presented at Neutrino 2002, Munich, Germany, May 2002.

18. K. Nishikawa for the K2K Collaboration, "Results from K2K", presented at Neutrino 2002, Munich, May 2002.

19. J. Imazato, "JHF Physics", Presented at ICFA Workshop on high intensity hadron beams, Fermilab, April 2002, Published in these proceedings.

20. G. Barenboim, A. De Gouvea, M. Szleper and M. Velasco, "Neutrino oscillations with a proton driver upgrade and an off axis detector: A case study", hep-ph/0204208 Apr. 2002. Also, M. Velasco, these proceedings.

21. G. Barenboim, etal., 'Physics potential at FNAL with stronger proton sources", hep-ph/0206025, Jun. 2002.

22. Also see various presentations from the "Workshop on new initiatives for the NuMI Neutrino Beam", Fermilab, May 2002, http://wwwnumi.fnal.gov/fnal_minos/new_initiatives/new_initiatives.html.

23. 'Letter of Intent for an Off-Axis Detector for the NuMI Beamline", June 2002, http://wwwnumi.fnal.gov/fnal_minos/new_initiatives/loi.html.

24. Report of the NuMI Proton Intensity Working Group, July 2002, http://hep.caltech.edu/ michael/numipiwg/.

25. Studies on a new proton driver for Fermilab. W. Chou and W. Foster editors. Reports in preparation. 